Kardiologe 2021 $15: 127-132$

https://doi.org/10.1007/s12181-021-00470-z

Angenommen: 9. Februar 2021

Online publiziert: 10. März 2021

๑) Deutsche Gesellschaft für Kardiologie -

Herz- und Kreislaufforschung e.V. Published by Springer Medizin Verlag GmbH, ein Teil von Springer Nature - all rights reserved 2021

Felix Mahfoud' $\cdot$ Michael Böhm ${ }^{1} \cdot$ Oliver Dörr ${ }^{2} \cdot$ Marcel Halbach $^{3} \cdot$ Ulrich Kintscher $^{4}$ für Nukleus der AG43: Arterielle Hypertonie der Deutschen Gesellschaft für Kardiologie

${ }^{1}$ Klinik für Innere Medizin III, Kardiologie Angiologie und Internistische Intensivmedizin, Universitätsklinikum des Saarlandes, Homburg/Saar, Deutschland

${ }^{2}$ Medizinische Klinik I, Kardiologie und Angiologie, Universitätsklinikum Gießen, Gießen, Deutschland

${ }^{3}$ Klinik III für Innere Medizin, Uniklinik Köln, Köln, Deutschland

${ }^{4}$ Institut für Pharmakologie, Center for Cardiovascular Research, Charité - Universitätsmedizin Berlin, Berlin, Deutschland

\title{
Arterielle Hypertonie - Was war 2020 wichtig?
}

nellen Verfahren zur Blutdruckbehandlung, die wir besprechen wollen.

Die arterielle Hypertonie ist immer noch der bedeutendste Risikofaktor für kardiovaskuläre Erkrankungen und damit einhergehende Behinderungen weltweit $[6,15]$. In Deutschland ist etwa jeder dritte Erwachsene von der Erkrankung betroffen [15, 25]. Die Prävalenz ist stark altersabhängig, sodass bei den 65- bis 79-Jährigen sogar $71 \%$ erkrankt sind $[15,25]$. Die Behandlungshäufigkeit hat in den letzten Jahren erfreulicherweise zugenommen, wobei immer noch ca. $30 \%$ der behandelten Patienten*innen nicht kontrolliert sind, d.h. unter Therapie nicht den entsprechenden Zielblutdruckbereich erreichen $[15,25]$. Neueste Daten aus der EUROASPIRE V-Studie weisen sogar auf einen Anteil von $\mathbf{4 2} \%$ an Patienten*innen mit unkontrolliertem Blutdruck ( $>140 / 90 \mathrm{~mm} \mathrm{Hg}$ ) in der Sekundärprävention hin $[15,17]$. Die Behandlungsstrategien für die arterielle Hypertonie müssen also unbedingt weiterentwickelt und verbessert werden.

Die COVID-19-Pandemie hat auch die arterielle Hypertonie und deren Behandlung im Jahr 2020 in den Fokus gerückt, und wir werden aktuelle Aspekte zu diesem Thema besprechen. Zusätzlich gab es weitere wichtige Studien und Diskussionsbeiträge unter anderem zur Chronotherapie und zu den neuen interventio-

\section{Arterielle Hypertonie und COVID-19}

Das Alter der Patienten*innen ist ein bedeutsamer Risikofaktor für die Schwere von COVID-19 einschließlich der Behandlung auf einer Intensivstation, einer mechanischen Beatmung und Tod $[9,10]$. Zusätzlich hat sich gezeigt, dass auch das Vorhandensein eines Bluthochdruckes mit einem schwereren COVID19-Krankheitsverlauf assoziiert ist. Es sollte jedoch berücksichtigt werden, dass einige dieser Assoziationen auch durch die höhere Prävalenz der arteriellen Hypertonie bei Älteren beeinflusst worden sind [8-10]. Dahingegen ist die Evidenz, dass Patienten*innen mit arterieller Hypertonie ein erhöhtes SARS-CoV-2Infektionsrisiko haben, eher schwach [10]. Mechanistisch konnte kürzlich in einer Studie an 48 Patienten*innen mittels Single-Cell-Transcriptomic-Profiling nachgewiesen werden, dass die arterielle Hypertonie eine spezifische immunologische Reaktion bei COVID19 auslöst, die mit einer verstärkten Entzündungsreaktion einhergeht [33]. Diese Prozesse könnten eine Ursache für den schwereren Krankheitsverlauf bei arterieller Hypertonie darstellen.

Seit Beginn der Pandemie wurde intensiv über die Sicherheit der antihypertensiven Behandlung mit pharmakologischen Blockern des Renin-Angioten-
sin-Systems (RAS), ACE(AngiotensinConverting-Enzym)-Hemmer und Angiotensin-Typ-1-Rezeptorblocker (ARB) diskutiert. Es ist bekannt, dass die medikamentöse Therapie mit diesen Substanzen wesentlich zur Reduktion der Morbidität und Mortalität von Patienten*innen mit arterieller Hypertonie beiträgt [35]. Eines der wichtigsten RAS-modulierenden Enzyme, ACE2, hat während der SARS-CoV-2-Pandemie zunehmend an Aufmerksamkeit gewonnen, da es nicht nur Angiotensin(ANG) II in den alternativen RAS-Metaboliten ANG 1-7 umwandelt, sondern auch als zellulärer Eintrittsrezeptor für SARS-CoV-2 fungiert (• Abb. 1; [16, 34]). ACE-Hemmer und ARBs können zu einer Hochregulierung der ACE2-Expression/Aktivität führen und wurden daher zu Beginn der SARS-CoV-2-Pandemie mit einem verschlechterten Outcome bei COVID19-Patienten in Verbindung gebracht (• Abb. 1; [16, 34]).

\section{》) Bluthochdruck ist mit einem schwereren COVID-19- Krankheitsverlauf assoziiert}

Inzwischen haben jedoch mehrere große Beobachtungsstudien beweisen können, dass weder das Risiko für eine SARSCoV-2-Infektion noch der Schweregrad der COVID-19-Erkrankung durch ACE-Hemmer oder ARBs negativ beeinflusst wird [23, 30, 31]. Hinsichtlich des Zusammenhangs zwischen RAS- 


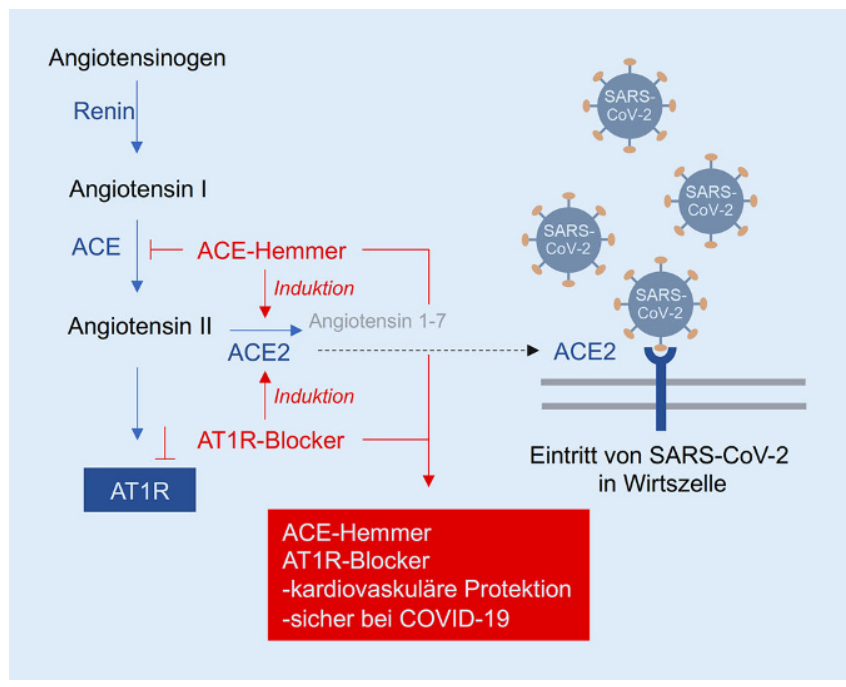

Abb. $1<$ Pharmakologische Blocker des Renin-Angiotensin-Systems und Interaktion mit SARS-CoV-2Rezeptor: ACE2 (Angiotensin-Converting-Enzym 2). AT1R Angiotensin Typ 1 Rezeptor
Blockern und Schweregrad des Verlaufs konnte kürzlich sogar in 2 randomisierten Studien dargelegt werden, dass weder ACE-Hemmer noch ARBs den COVID-19-Verlauf negativ beeinflussen. Die REPLACE-COVID-Studie randomisierte 152 Patienten*innen, die mit COVID-19 stationär behandelt wurden und mit RAS-Blockern vorbehandelt waren, entweder auf einen Arm, in dem die ACE-Hemmer/ARB-Therapie fortgesetzt wurde, oder auf den Arm, in dem diese Therapie abgesetzt wurde [5]. Es ergab sich kein signifikanter Unterschied in den beiden Armen bezüglich des primären Endpunktes, der einen „rank score for clinical outcome during hospitalization" mit mehreren klinischen Parametern (Tage bis zum Tod, Dauer der Beatmung, Dauer der Dialyse, SOFA-Score) umfasste [5]. Zu vergleichbaren Ergebnissen kam die BRACECORONA-Studie, die in diesem Jahr publiziert wurde [21]. Hier wurden 659 COVID-19-Patienten*innen eingeschlossen und ebenfalls auf Fortführung bzw. Beendigung der ACE/ARB-Therapie randomisiert [21]. Auch hier zeigte sich kein signifikanter Unterschied zwischen den Studienarmen bezüglich des primären Endpunktes, Dauer des Überlebens und Entlassung über einen 30Tage-Zeitraum (• Abb. 2; [21]).

Zusammenfassend kann also festgehalten werden, dass ACE-Hemmer und ARBs weder mit einem erhöhten SARS-CoV-2-Infektionsrisiko noch mit einem schwereren Krankheitsverlauf von
COVID-19 assoziiert sind. Es ist eher davon auszugehen, dass der Einsatz dieser Medikamente auch während COVID19 langfristig eher zu einem Benefit, vermittelt über deren kardiovaskulär protektive Effekte, führt.

\section{Antihypertensive Chrono- therapie}

Physiologische, tageszeitabhängige Variationen mit einem morgendlichen Blutdruckanstieg und einem nächtlichen Blutdruckabfall finden bei den aktuellen Diagnose- und Therapieempfehlungen Berücksichtigung [35]. Die JAMP(Japan Ambulatory Blood Pressure Monitoring Prospective)-Studie, an der zwischen 2009 und 2017 insgesamt 6359 Patienten im medianen Alter von 68 Jahren teilnahmen, bei denen wenigstens ein kardiometabolischer Risikofaktor vorlag, untersuchte die prognostische Bedeutung des nächtlichen Blutdrucks und des Blutdruckprofils [13]. Zum Zeitpunkt der Langzeitmessung hatte keiner der Patienten eine symptomatische Erkrankung. Während der Nachbeobachtung von 4,5 Jahren erlitten 119 Patienten einen Schlaganfall, 99 ein koronares Ereignis und 88 eine Herzinsuffizienz. Interessanterweise hatten die Praxisblutdruckwerte keine prognostische Bedeutung, wohl aber die nächtlichen Blutdruckwerte. Ein Anstieg des systolischen Blutdrucks um $20 \mathrm{~mm} \mathrm{Hg}$ war mit einem Anstieg von atherosklerotischen kardiovaskulären
Ereignissen um $18 \%$ verbunden (Hazard Ratio 1,18; $95 \%$-KI 1,02-1,37). Das Risiko für eine chronische Herzinsuffizienz lag mit $25 \%$ darüber (Hazard Ratio 1,25; $95 \%$-KI 1,00-1,55).

Auch vor dem Hintergrund der prognostischen Relevanz des nächtlichen Blutdrucks wird seit einigen Jahren die sog. Chronotherapie untersucht, bei der die antihypertensive Therapie bevorzugt am Abend verabreicht wird. Im letzten Jahr wurde im European Heart Journal die HYGIA-Studie veröffentlicht, die $\mathrm{zu}$ einigen Diskussionen führte [11]. In dieser Multicenter-PROBE(„prospective, randomized, open-label, blinded endpoint")Design-Studie wurden 19.084 Patienten ${ }^{\star}$ innen mit arterieller Hypertonie in 40 spanischen Hausarztpraxen von 292 Ärzten $^{\star}$ innen rekrutiert [11]. Die Patienten*innen wurden 1:1 randomisiert auf die abendliche Einnahme von $\geq 1$ blutdrucksenkenden Medikament (Bedtime-Regimen: $n=9552$ ) oder die morgendliche Medikamenteneinnahme (Awakening-Treatment-Regimen: $n=9532$ ). Nach einem Median-Followup von 6,3 Jahren zeigte sich eine deutliche Reduktion des primären kardiovaskulären Endpunktes aus Myokardinfarkt, koronarer Revaskularisation, Herzinsuffizienz, ischämischem oder hämorrhagischem Schlaganfall oder kardiovaskulärem Tod in der Gruppe mit abendlicher Medikamentengabe $(\mathrm{HR}=0,55$ [95\%CI [Konfidenzintervall] 0,50-0,061], $p>0,001)$. Der Vorteil der abendlichen Gabe zeigte sich auch in allen Subgruppen. Es wurde daher geschlussfolgert, dass eine generelle abendliche Tabletteneinnahme das kardiovaskuläre Risiko von Patienten ${ }^{\star}$ innen mit arterieller Hypertonie reduzieren kann. In der Studie wurde interessanterweise eine verlängerte Langzeitblutdruckmessung über $48 \mathrm{~h}$ durchgeführt. Hier zeigte sich ein um $3,3 \mathrm{~mm} \mathrm{Hg}$ niedrigerer nächtlicher systolischer und ein um 1,6 $\mathrm{mm} \mathrm{Hg}$ niedrigerer nächtlicher diastolischer Blutdruck in der Bedtime-Regime-Gruppe im Vergleich zur morgendlichen Einnahme [11]. Die Tageswerte für den systolischen und diastolischen Blutdruck waren nicht signifikant unterschiedlich [11]. 
Mit dem Wissen, dass ein exzessiver nächtlicher Blutdruckabfall, sog. Overoder Extreme-Dipping, mit einem erhöhten Risiko für ischämische Ereignisse bei Patienten*innen mit koronarer Herzerkrankung assoziiert ist und das Risiko für stille zerebrale Infarkte erhöht wird, sind diese Ergebnisse sehr kontrovers diskutiert worden [14, 18, 28]. Zusätzlich konnte in der Vergangenheit in Studien, in denen die nächtliche mit der morgendlichen Medikamenteneinnahme verglichen wurde, kein signifikanter Unterschied in ABDM(ambulantes 24-h-Blutdruckmonitoring)-Tages- oder -Nachtwerten detektiert werden [29]. In der genaueren Analyse der HYGIA-Studie wurden weitere Limitationen angesprochen einschließlich einer auffällig niedrigen Drop-out-Rate trotz der Verwendung des den Patienten belastenden 48$\mathrm{h}-\mathrm{ABDM}$ und Versäumnissen in der Befolgung allgemeingültiger Regeln bei der Durchführung von randomisierten klinischen Studien, wie z. B. die vorzeigte Beendigung der Studie bei eindeutigem Unterschied zwischen den Gruppen [18]. Aufgrund der genannten Limitationen kann eine generelle abendliche Gabe der antihypertensiven Medikation nicht empfohlen werden [18]. Hinzu kommt, dass die Medikamentenadhärenz außerhalb klinischer Studien möglicherweise bei morgendlicher Einnahme höher ist [27].

\section{Renale Denervation 2020}

Eine chronische Überaktivität des sympathischen Nervensystems ist ein wichtiger pathophysiologischer Faktor bei der Entstehung und Progression der arteriellen Hypertonie und begleitender Organschäden $[20,26]$. Hier spielen die efferenten und afferenten sympathischen Nervenfasern der Niere eine besondere Rolle [7, 20]. Die Stimulation der renalen sympathischen Nerven kann über unterschiedliche Mechanismen zur systemischen Blutdruckerhöhung beitragen [20]. Die katheterbasierte Ablation der sympathischen Nervenfasern im Bereich der Nierenarterie wurde vor 11 Jahren erstmals bei Patienten mit therapieresistenter Hypertonie durchgeführt [19]. Nach der Publikation einiger positiver

Kardiologe 2021 · 15:127-132 https://doi.org/10.1007/s12181-021-00470-z

(c) Deutsche Gesellschaft für Kardiologie - Herz- und Kreislaufforschung e.V. Published by Springer Medizin Verlag GmbH, ein Teil von Springer Nature - all rights reserved 2021

F. Mahfoud · M. Böhm · O. Dörr · M. Halbach · U. Kintscher für Nukleus der AG43: Arterielle Hypertonie der Deutschen Gesellschaft für Kardiologie

\section{Arterielle Hypertonie - Was war 2020 wichtig?}

\section{Zusammenfassung}

Die arterielle Hypertonie bleibt weltweit der prävalenteste Risikofaktor für kardiovaskuläre Erkrankungen und damit einhergehende Behinderungen. Auch im Bereich der arteriellen Hypertonie bestimmte die COVID-19-Pandemie einen Teil der wissenschaftlichen Debatte. Die arterielle Hypertonie ist mit einem schwereren Krankheitsverlauf von COVID-19 assoziiert, wohingegen das SARS-CoV-2-Infektionsrisiko bei Hypertonikern nicht erhöht zu sein scheint. Nach aktueller Datenlage ist die Therapie mit ACE(Angiotensin-ConvertingEnzym)-Hemmer und Angiotensin-Typ-1Rezeptorblocker weder mit einem erhöhten SARS-CoV-2-Infektionsrisiko noch mit einem schwereren Krankheitsverlauf von COVID-19 verbunden. Eine Studie zur antihypertensiven Chronotherapie bestimmte den wissenschaftlichen Diskurs zur medikamentösen Therapie der Hypertonie. Die HYGIA-Studie kam zu der Schlussfolgerung, dass eine abendliche Medikamenteneinnahme das kardiovaskuläre Risiko von Patienten*innen mit arterieller Hypertonie reduziert. Aufgrund einiger Limitationen ist die Datenlage aktuell unzureichend, um eine routinemäßige nächtliche Gabe von antihypertensiven Medikamenten zu empfehlen. Wir werden einige der Aspekte diskutieren. Ein weiterer wissenschaftlicher Schwerpunkt lag auf den neuen Studien zur renalen Denervation. Hier kann man zusammenfassen, dass nach neuer Studienlage die katheterbasierte renale Denervation ein effektives und sicheres Verfahren zur Behandlung der arteriellen Hypertonie darstellt, das sich als alternatives Verfahren zur medikamentösen Blutdrucksenkung weiter etablieren könnte.

\section{Schlüsselwörter}

COVID-19 · ACE-Hemmer · Renale Denervation · Chronotherapie · SARS-CoV-2

\section{Arterial hypertension-What was important in 2020?}

\section{Abstract}

Arterial hypertension remains the most significant risk factor for cardiovascular disease and associated disability worldwide. In the field of arterial hypertension the coronavirus disease 2019 (COVID-19) pandemic also determined major parts of the scientific debate. Arterial hypertension is associated with a severe course of COVID19 , whereas risk of infection by severe acute respiratory syndrome coronavirus 2 (SARSCoV-2) does not appear to be increased in hypertensive individuals. According to current data, treatment with angiotensin-converting enzyme (ACE) inhibitors and angiotensin type 1 receptor blockers is not associated with an increased risk of SARS-CoV-2 infection or with a more severe course of COVID-19. A study on antihypertensive chronotherapy determined the scientific discourse on the medication treatment of hypertension. The
HYGIA study concluded that bedtime medication reduces the cardiovascular risk in patients with arterial hypertension. Due to some study limitations, routine administration of bedtime antihypertensive medication cannot be recommended. Some of the reasons are discussed herein. Another scientific focus was on new renal denervation studies. Here, one can summarize that according to novel evidence, catheter-based renal denervation is an effective and safe procedure for the treatment of arterial hypertension, which could become established as an alternative to pharmaceutical blood pressure reduction in the near future.

\section{Keywords}

COVID-19 - ACE inhibitor - Renal denervation · Chronotherapy · SARS-CoV-2 


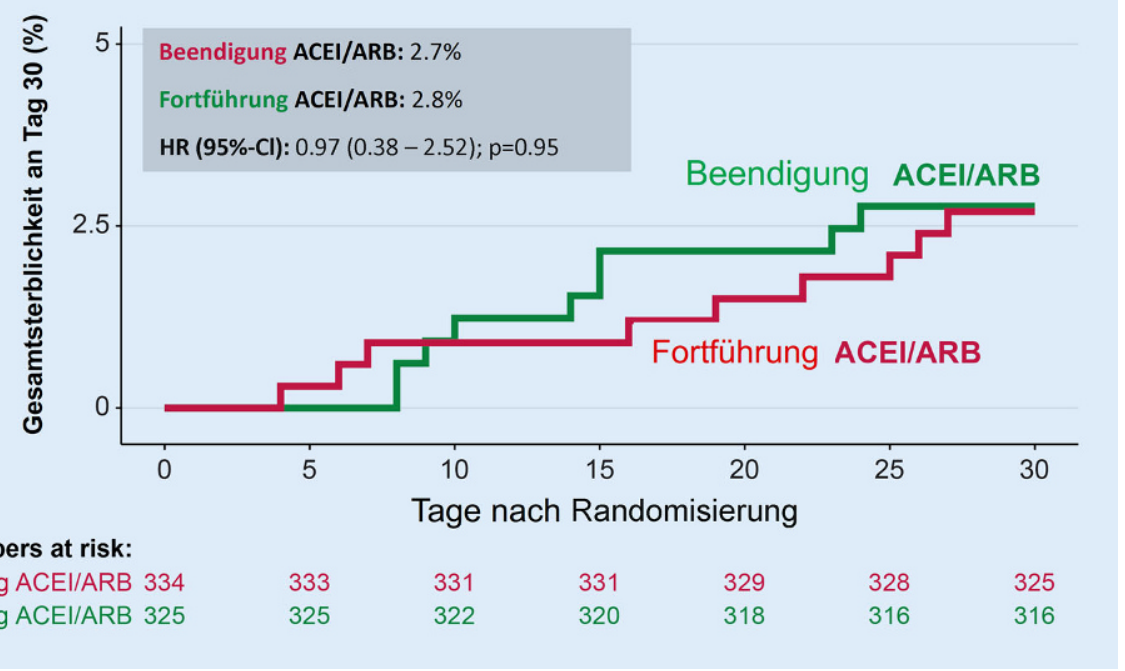

Abb. 2 \ Randomisierte klinische Studie (BRACECORONA) - Vergleich der Beendigung vs. Fortführung einer ACE(Angiotensin-Converting-Enzym)-Hemmer(ACEI)/Angiotensin-Typ-1-Rezeptor-Blocker(ARB)-Therapie bei hospitalisierten COVID-19-Erkrankten. Gesamtsterblichkeit an Tag 30. HR Hazard Ratio, CI Konfidenzintervall [21]

Studien zeigte sich in der randomisierten, scheinkontrollierten SYMPLICITY HTN-3-Studie, dass die renale Denervation keinen Vorteil gegenüber einer Scheinprozedurhinsichtlich derSenkung des systolischen Blutdruckes hatte [2]. Die Ergebnisse und mögliche Limitationen der Studien wurden sehr kontrovers diskutiert.

Durch eine kontinuierliche Verbesserung des Studiendesign und der interventionellen Methodik ist es jetzt in den letzten 3 Jahren gelungen, eine zweite Generation von Studien zur renalen Denervation durchzuführen und zu publizieren. Drei randomisierte, shamkontrollierte klinische Studien SPYRAL HTN-OFF MED, SPYRAL HTN-ON MED und RADIANCE-HTN SOLO konnten konsistent nachweisen, dass die radiofrequenz- oder ultraschallbasierte renale Denervation zu einer klinisch relevanten Reduktion des Praxisblutdruckes und des ambulanten 24-h-Blutdruckes (ABDM) führt $[1,3,12,32]$. Sowohl nach 2 bis 3 Monaten als auch nach 6 Monaten resultierte die renale Denervation in einer Senkung des systolischen Praxisblutdruckes $[1,3,12,32]$. Diese Daten belegen eindeutig die Wirksamkeit des Verfahrens bei behandelten und unbehandelten Patienten ${ }^{*}$ innen mit milder bis moderater arterieller Hypertonie (• Abb. 3). Zusätzlich konnten alle Studi- en die klinische Sicherheit der Methode belegen.

Es stellt sich also nun die Frage, wie und wann diese Behandlungsmethode in die klinische Routine integriert wird? Die aktuellen Leitlinien der European Society of Cardiology (ESC) und der europäischen Hypertoniegesellschaft (ESH) von 2018 berücksichtigen die genannten neuen Studien nicht, da sie zu dem Zeitpunkt der Leitlinienerstellung noch nicht veröffentlicht waren [35]. Vor dem Hintergrund der nun verfügbaren $\mathrm{Da}$ ten können aber Rückschlüsse auf die zukünftige klinische Anwendung der interventionellen renalen Denervation gezogen werden.

Wichtig erscheint hier eine adäquate Patientenselektion. Obwohl die Patientenpopulationen in den neuen Studien keine Patienten*innen mit schwerer, therapieresistenter Hypertonie inkludierten, scheint die interventionelle antihypertensive Therapie gerade für $\mathrm{Pa}$ tienten ${ }^{\star}$ innen mit einem schwer einstellbaren arteriellen Hypertonus und einer medikamentösen Therapieresistenz eine geeignete zusätzliche Behandlungsalternative darzustellen $[4,24]$. Hierbei ist jedoch zu beachten, dass im Vorfeld eine sekundäre arterielle Hypertonie auszuschließen ist und dass eine umfassende Untersuchung der Therapieadhärenz der Patienten*innen erfolgen sollte $[4,24]$.
Auch für Patienten*innen, die strikt gegen eine medikamentöse Behandlung sind oder unter starken Nebenwirkungen leiden, könnte die renale Denervation erwogen werden. Die Einbeziehung der Patienten*innen in die Therapieentscheidung mittels eines "Shared decision making"-Ansatzes sollte ebenfalls berücksichtigt werden $[4,24]$. Weitere Patientengruppen, die von einer renalen Denervation profitieren könnten, gilt es, in zukünftigen Studien zu identifizieren. Erst kürzlich konnte beispielsweise in einer Analyse des weltweit größten Denervationsregisters (Global SYMPLICITY-Register) bestätigt werden, dass die renale Denervation auch bei Patienten*innen mit hohem kardiovaskulärem Risiko effektiv ist [22]. Es bleibt außerdem festzuhalten, dass die interventionelle renale Denervation derzeitig und in nächster Zukunft ausschließlich von spezialisierten Zentren durchgeführt werden sollte $[4,24]$.

In naher Zukunft werden weitere wichtige Studienergebnisse zur renalen Denervation erwartet. Diese umfassen sowohl neue Studien zur ultraschallbasierten Denervation bei Patienten ${ }^{*}$ innen mit therapieresistenter Hypertonie unter 3-fach Fixkombination (RADIANCE HTN-TRIO, REQUIRE, RADIANCE II) als auch Studien zu neuen interventionellen Verfahren wie dem Peregri- 


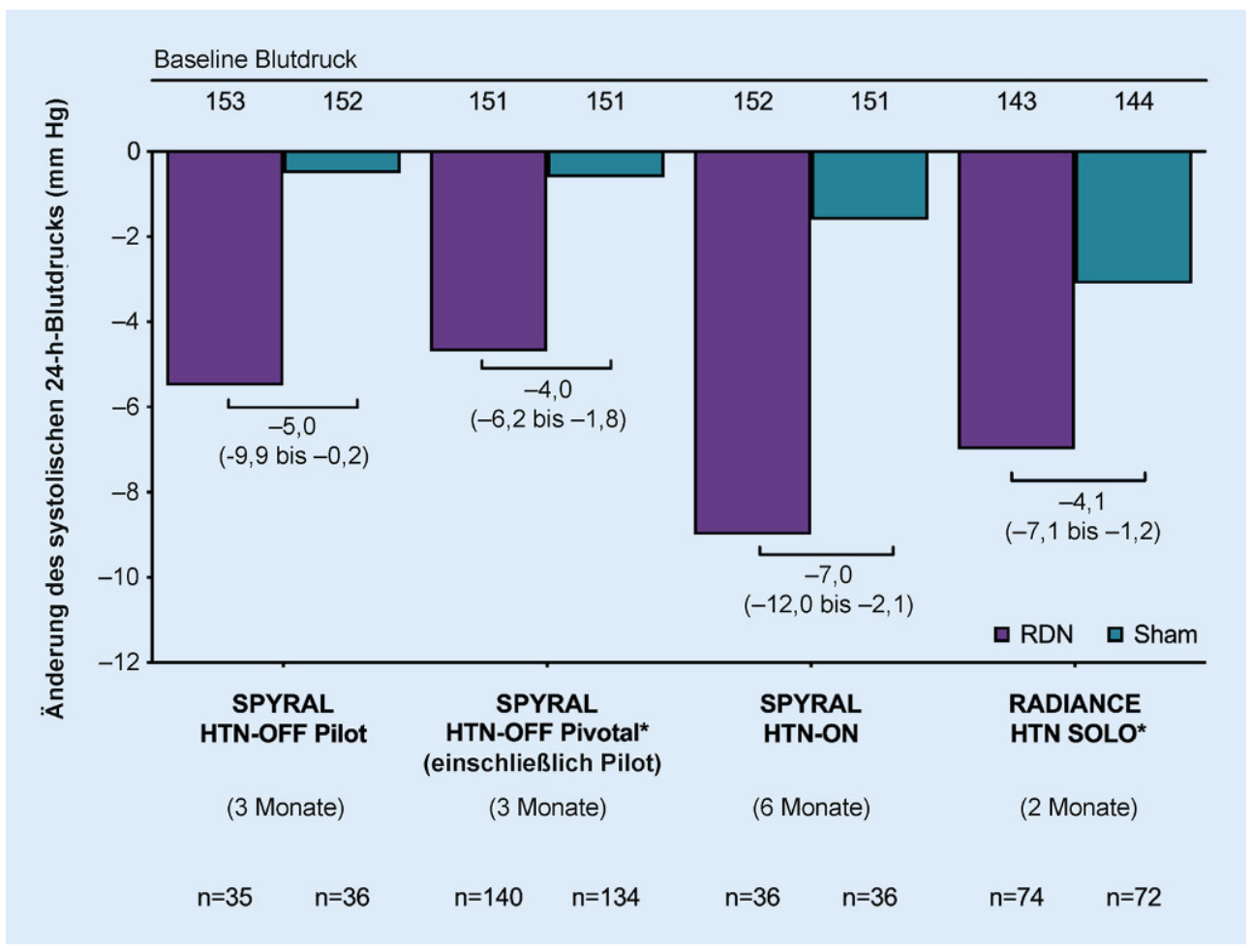

Abb. 3 \ Änderung des 24-h-Blutdrucks in den neuen Studien zur renalen Denervation. Sternchen Gepowerte Studien. Daten werden als Mittelwert $(\mathrm{mm} \mathrm{Hg})$ und $95 \%$-Konfidenzintervall dargestellt. RDN Renale Denervation

ne-Kathetersystem (Ablative Solutions, Wakefield, MA, USA), bei dem durch Mikronadeln purer Alkohol in den perivaskulären Raum der Nierenarterien appliziert wird (TARGT B I, TARGET BP OFF-MED) [20].

\section{》) Die renale Denerva- \\ tion ist auch bei hohem kardiovaskulärem Risiko effektiv}

Vor dem Hintergrund, dass eine erhöhte sympathische Nervenaktivität auch bei anderen kardiovaskulären Erkrankungen bedeutsam ist, wird die renale Denervation auch in anderen Indikationen getestet, wie beispielsweise bei der chronischen Nierenerkrankung (RDN-CKD), bei Herzinsuffizienz mit reduzierter Ejektionsfraktion (RE-ADAPT-HF) oder bei Vorhofflimmern (SYMPLICITY-HF).

Man kann also zusammenfassen, dass die interventionelle renale Denervation nach neuer Studienlage nachweislich den Blutdruck senken kann und sicher ist. Es ist anzunehmen, dass sich die renale Denervation zunehmend als eine weitere, alternative Behandlungsoption bei ausgewählten Patienten etablieren wird.

\section{Fazit für die Praxis}

- Die arterielle Hypertonie ist mit einem schwereren Krankheitsverlauf von COVID-19 assoziiert.

- Das SARS-CoV-2-Infektionsrisiko scheint bei Hypertonikern nicht erhöht zu sein.

- Die Therapie mit ACE(AngiotensinConverting-Enzym)-Hemmer und Angiotensin-Typ-1-Rezeptorblocker geht weder mit einem erhöhten SARS-CoV-2-Infektionsrisiko noch mit einem schwereren Krankheitsverlauf von COVID-19 einher.

- Trotz der Ergebnisse der HYGIA-Studie, in der die abendliche Einnahme von blutdrucksenkenden Medikamenten das kardiovaskuläre Risiko im Vergleich zur morgendlichen Einnahme reduzierte, wird aktuell von der routinemäßigen abendlichen Einnahme abgeraten.

- Die katheterbasierte renale Denervation stellt ein effektives und sicheres Verfahren zur Behandlung der arteriellen Hypertonie dar, deren Wirksamkeit durch eine zweite Generation sham-kontrollierter, klinischer Studien bestätigt werden konnte. Somit kann die renale Denervation als eine Alternative bzw. in Ergänzung zur medikamentösen Therapie bei ausgewählten Patienten in Betracht gezogen werden.

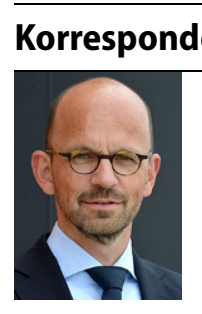

Ulrich Kintscher Institut für Pharmakologie, Center for Cardiovascular Research, Charité Universitätsmedizin Berlin Hessische Str. 3-4, 10115 Berlin, Deutschland ulrich.kintscher@charite.de

\section{Einhaltung ethischer Richtlinien}

Interessenkonflikt. F. Mahfoud gibt an, Forschungsunterstützung und Vortragshonorare von Medtronic, Recor, Berlin Chemie und Boehringer Ingelheim erhalten zu haben und durch die Deutsche Forschungsgemeinschaft (SFB-TRR219) unterstützt zu werden. M. Böhm gibtan, Vortragshonorare von Abbot, Amgen, AstraZeneca, Bayer, Boehringer Ingelheim, Medtronic, 
Novartis, Servier und Vifor erhalten zu haben. O. Dör gibt an, Vortragshonorare von Medtronic erhalten zu haben. M. Halbach hat Vortragshonorare von Abbott, AstraZeneca, Bayer, BMS/Pfizer, CVRx, Daiichi-Sankyo, Gilead, Medtronic, MSD, Sanofi-Aventis, Vifor und ViiV erhalten sowie Forschungsförderung durch CVRx. U. Kintscher hat Vortrags- und/oder Beraterhonorare von Bayer, Berlin Chemie, Boehringer Ingelheim, Daiichi Sankyo, Novartis, Sanofi und Servier erhalten. Forschungsarbeiten der Arbeitsgruppe Kintscher wurden von Bayer unterstützt.

Für diesen Beitrag wurden von den Autoren keine Studien an Menschen oder Tieren durchgeführt. Für die aufgeführten Studien gelten die jeweils dort angegebenen ethischen Richtlinien.

\section{Literatur}

1. Azizi M, Schmieder RE, Mahfoud F et al (2018) Endovascular ultrasound renal denervation to treat hypertension (RADIANCE-HTN SOLO): a multicentre, international, single-blind, randomised, sham-controlled trial. Lancet 391:2335-2345

2. Bhatt DL, Kandzari DE, O'neill WW et al (2014) A controlled trial of renal denervation for resistant hypertension. N Engl J Med. https://doi.org/10. 1056/NEJMoa1402670

3. Bohm M, Kario K, Kandzari DE etal (2020) Efficacy of catheter-based renal denervation in the absence of antihypertensive medications (SPYRAL HTN-OFF MED Pivotal): a multicentre, randomised, shamcontrolled trial. Lancet 395:1444-1451

4. Bruno RM, Taddei S, Borghi C et al (2020) Italian Society of Arterial Hypertension (SIIA) position paper on the role of renal denervation in the management of the difficult-to-treat hypertensive patient. High Blood Press Cardiovasc Prev 27:109-117

5. Cohen JB, HanffTC, William P et al (2021) Continuation versus discontinuation of renin-angiotensin system inhibitors in patients admitted to hospital with COVID-19: a prospective, randomised, openlabel trial. Lancet Respir Med. https://doi.org/10. 1016/S2213-2600(20)30558-0

6. Collaborators GBDRF (2016) Global, regional, and national comparative risk assessment of 79 behavioural, environmental and occupational, and metabolic risks or clusters of risks, 1990-2015: a systematic analysis for the Global Burden of Disease Study 2015. Lancet 388:1659-1724

7. Dibona GF (2003) Neural control of the kidney: past, present, and future. Hypertension 41:621-624

8. Gao C, Cai Y, Zhang Ket al (2020) Association of hypertension and antihypertensive treatment with COVID-19 mortality: a retrospective observational study. Eur Heart J 41:2058-2066

9. Guan WJ, Ni ZY, Hu Y et al (2020) Clinical characteristics of coronavirus disease 2019 in China. NEngl J Med 382:1708-1720

10. Guzik TJ, Mohiddin SA, Dimarco A et al (2020) COVID-19 and the cardiovascular system: implications for risk assessment, diagnosis, and treatment options. Cardiovasc Res 116:1666-1687

11. Hermida RC, Crespo JJ, Dominguez-Sardina M et al (2020) Bedtime hypertension treatment improves cardiovascular risk reduction: the Hygia Chronotherapy Trial. Eur Heart J 41:4565-4576

12. Kandzari DE, Bohm M, Mahfoud F et al (2018) Effect of renal denervation on blood pressure in the presence of antihypertensive drugs: 6-month efficacy and safety results from the SPYRAL HTN-
ONMED proof-of-concept randomised trial. Lancet 391:2346-2355

13. Kario K, Hoshide S, Mizuno Het al (2020) Nighttime blood pressure phenotype and cardiovascular prognosis: practitioner-based nationwide JAMP study. Circulation 142:1810-1820

14. Kario K, Pickering TG, Matsuo T et al (2001) Stroke prognosis and abnormal nocturnal blood pressure falls in older hypertensives. Hypertension 38:852-857

15. Kintscher U (2020) Update Hypertonie: Ab wann und wie behandeln? CME 17:9-17

16. Kintscher $U$, Slagman A, Domenig $O$ et al (2020) Plasma angiotensin peptide profiling and ACE (angiotensin-converting enzyme)2 activity in COVID-19 patients treated with pharmacological blockers of the renin-angiotensin system. Hypertension 76:e34-e36

17. Kotseva K, De Backer G, De Bacquer D et al (2019) Lifestyle and impact on cardiovascular risk factor control in coronary patients across 27 countries: results from the European Society of Cardiology ESC-EORP EUROASPIRE V registry. Eur JPrev Cardio 26:824-835

18. Kreutz R, Kjeldsen SE, Burnier M et al (2020) Disregard the reported data from the HYGIA project: blood pressure medication not to be routinely dosed at bedtime. J Hypertens 38:2144-2145

19. Krum H, Schlaich M, Whitbourn R et al (2009) Catheter-based renal sympathetic denervation for resistant hypertension: a multicentre safety and proof-of-principle cohort study. Lancet 373:1275-1281

20. Lauder L, Azizi M, Kirtane AJ et al (2020) Devicebased therapies for arterial hypertension. Nat Rev Cardiol 17:614-628

21. Lopes RD, Macedo AVS, De Barros ESPGM et al (2021) Effect of discontinuing vs continuing angiotensin-converting enzyme inhibitors and angiotensin II receptor blockers on days alive and out of the hospital in patients admitted with COVID-19: a randomized clinical trial. JAMA 325:254-264

22. Mahfoud F, Mancia G, Schmieder R et al (2020) Renal denervation in high-risk patients with hypertension. JAm Coll Cardiol 75:2879-2888

23. Mancia G, Rea F, Ludergnani M et al (2020) Reninangiotensin-aldosterone system blockers and the risk of Covid-19. N Engl J Med. https://doi.org/10 1056/NEJMoa2006923

24. Millenaar D, Emrich I, Mahfoud F (2020) Renal denervation in daily practice: if so, how? High Blood Press Cardiovasc Prev 27:267-270

25. Neuhauser HK, Adler C, Rosario AS et al (2015) Hypertension prevalence, awareness, treatment and control in Germany 1998 and 2008-11. J Hum Hypertens 29:247-253

26. Parati G, Esler M (2012) The human sympathetic nervous system: its relevance in hypertension and heart failure. Eur Heart J 33:1058-1066

27. Perry LA, Surowiec SM, Danso DA et al (2019) Evaluation of administration time and adherence rates of morning vs. bedtime dosing of antihypertensive medications. JContemp Pharm Pract 66:11-16

28. Pierdomenico SD, Bucci A, Costantini F et al (1998) Circadian blood pressure changes and myocardial ischemia in hypertensive patients with coronary artery disease. J Am Coll Cardiol 31:1627-1634

29. Rahman M, Greene T, Phillips RA et al (2013) A trial of 2 strategies to reduce nocturnal blood pressure in blacks with chronic kidney disease. Hypertension 61:82-88
30. Reynolds HR, Adhikari S, Pulgarin C et al (2020) Renin-angiotensin-aldosterone system inhibitors and risk of Covid-19. N Engl J Med. https://doi.org/ 10.1056/NEJMoa2008975

31. Semenzato L, Botton J, Drouin J et al (2021) Antihypertensive drugs and COVID-19 risk: a cohort study of 2 million hypertensive patients. Hypertension. https://doi.org/10.1161/HYPERTENSIONAHA.120. 16314

32. Townsend RR, Mahfoud F, Kandzari DE et al (2017) Catheter-based renal denervation in patients with uncontrolled hypertension in the absence of antihypertensive medications (SPYRAL HTN-OFF MED): a randomised, sham-controlled, proof-ofconcept trial. Lancet 390:2160-2170

33. Trump S, Lukassen S, Anker MS et al (2020) Hypertension delays viral clearance and exacerbates airway hyperinflammation in patients with COVID-19. Nat Biotechnol. https://doi.org/10. 1038/s41587-020-00796-1

34. Vaduganathan $M$, Vardeny 0 , Michel $T$ et al (2020) Renin-angiotensin-aldosterone system inhibitors in patients with Covid-19. N Engl J Med 382:1653-1659

35. Williams B, Mancia G, Spiering W et al (2018) 2018 ESC/ESH Guidelines for the management of arterial hypertension. Eur Heart J 39:3021-3104 